\title{
WAVE FRONT SETS AND THE VISCOSITY METHOD
}

\author{
BY JOEL A. SMOLLER AND MICHAEL E. TAYLOR ${ }^{1}$ \\ Communicated by François Treves, August 8, 1972
}

Consider the initial value problem

$$
\frac{\partial}{\partial t} u+\sum_{j=1}^{n} A_{j}(x, t) \frac{\partial}{\partial x_{j}} u=0, \quad u(x, 0)=\Phi(x) .
$$

Here $A_{j}(x, t)$ are smooth $k \times k$ matrix-valued functions on $R^{n} \times R$, constant for large $|x|+|t|$, and $u_{0} \in H^{s}\left(R^{n}\right)$ for some $s \in R$. We assume that (1) is strictly hyperbolic in the sense that $\sum_{1}^{n} \xi_{j} A_{j}$ has real and distinct eigenvalues for all $\xi=\left(\xi_{1}, \ldots, \xi_{n}\right) \neq 0$. The solution to this problem is a weak, i.e., distribution, limit of solutions $u_{\varepsilon}$ of the problem

$$
\frac{\partial}{\partial t} u_{\varepsilon}+\sum_{j=1}^{n} A_{j}(x, t) \frac{\partial}{\partial x_{j}} u_{\varepsilon}=\varepsilon B\left(x, D_{x}\right) u_{\varepsilon}, \quad u_{\varepsilon}(x, 0)=\Phi(x),
$$

where $\varepsilon>0$ and $B\left(x, D_{x}\right)$ is an elliptic operator of order two, whose principal symbol is a $k \times k$ matrix with positive eigenvalues ${ }^{2}$. In fact, this is the well-known technique of parabolic regularization, or the "viscosity" method.

Our interest in these problems arises from the study of systems of nonlinear conservation laws. In studying computer output of such systems, we observed that for certain viscosity matrices, suggested in [1], the solutions $u_{\varepsilon}$ were not converging to the correct solution, but were converging to a solution containing an extraneous shock wave. We discovered similar behavior for linear systems and it is this phenomenon which we investigate here.

It is known (see [3], [5]) that the singularities of the solution $u$ to (1) propagate along bicharacteristics through the singularities of the initial data $\Phi$. In our numerical experiments, for $n=1, k=2$, we took $\Phi$ to have a single singularity at the origin, and we knew that the singularity of the solution lay along precisely one bicharacteristic ray through the origin. However, we found that the approximate solutions seemed to be developing a singularity along the second bicharacteristic through the

AMS (MOS) subject classifications (1970). Primary 35B25, 35F10, 35K45.

${ }^{1}$ The authors acknowledge the partial support of grants AFOSR-69-1662 and NSF GP-18903, respectively.

${ }^{2}$ We could more generally handle the case where the eigenvalues have positive real parts. 
origin. This suggests two problems: look for examples of "illusory" singularities of the solution $u_{\varepsilon}$ to (2); and investigate whether such singularities only occur along bicharacteristics through singularities in $\Phi$. We outline our results here; complete proofs will be given elsewhere.

EXAmple 1. Let $n=2=k, A=\left(\begin{array}{ll}1 & 0 \\ 0 & 0\end{array}\right), B=\left(\begin{array}{ll}1 & 0 \\ 1 & 1\end{array}\right), U=(u, v)^{t}$, and consider the system

$$
\frac{\partial}{\partial t} U_{\varepsilon}+A \frac{\partial}{\partial x} U_{\varepsilon}=\varepsilon B \frac{\partial^{2}}{\partial x^{2}} U_{\varepsilon}, \quad \varepsilon>0
$$

with initial data $u=\delta, v \equiv 0$. The solution is given by the well-known formula

$$
U_{\varepsilon}(x, t)=\frac{1}{(2 \pi)^{1 / 2}} \int_{-\infty}^{\infty} e^{\Gamma(\varepsilon, \xi, t, x)} \hat{\Phi}(\xi) d \xi
$$

where $\Gamma=\left(i A \xi-\varepsilon B \xi^{2}\right) t+i x \xi$, and $\hat{\Phi}$ denotes the Fourier transform of $\Phi$, which in this case is the vector $(1,0)^{t}$. In order to evaluate this integral, we diagonalize $\Gamma$ : If $\lambda_{ \pm}$are the eigenvalues of $\Gamma$, and $\Delta=\operatorname{diag}\left(\lambda_{+}, \lambda_{-}\right)$, then $e^{\Gamma}=S e^{\Delta} S^{-1}$, where $S=\left(\begin{array}{cc}\theta & 0 \\ 1 & 1\end{array}\right), \theta=i \varepsilon \xi$.

One finds that

$$
\left(\begin{array}{l}
u_{\varepsilon}(x, t) \\
v_{\varepsilon}(x, t)
\end{array}\right)=\int_{-\infty}^{\infty}\left(\begin{array}{c}
e^{\lambda_{+}} \\
i x \xi\left(e^{\lambda_{+}}-e^{\lambda_{-}}\right)
\end{array}\right) d \xi,
$$

and a calculation yields

$$
v_{\varepsilon}(x, t)=\frac{2 x}{t} \frac{e^{-x^{2} / \varepsilon t}}{(\varepsilon t)^{1 / 2}}-\frac{2(x+t)}{t} \frac{e^{-(x+t)^{2} / \varepsilon t}}{(\varepsilon t)^{1 / 2}} .
$$

This sequence does indeed converge pointwise to zero as $\varepsilon \rightarrow 0$, but does not converge uniformly (in $x$ ) to zero. It is precisely this phenomenon which showed up in our numerical calculations. We thus see that great care must be taken in applying the viscosity method for numerical calculation of discontinuous solutions to initial value problems, in particular for nonlinear hyperbolic conservation laws.

EXAMPLE 2. If one gives up strict hyperbolicity then the $u_{\varepsilon}$ need not even converge pointwise to the correct solution. To see this, let $n=2=k$, let $A=$ identity, $B=\left(\begin{array}{ll}1 & 0 \\ 1 & 2\end{array}\right)$, and take the same data as in Example 1 . Calculations similar to those above show

$$
v_{\varepsilon}(x, t)=\frac{e^{-(x+t)^{2} / 2 \varepsilon t}}{(2 \varepsilon t)^{1 / 2}}-\frac{e^{-(x+t)^{2} / \varepsilon t}}{(\varepsilon t)^{1 / 2}},
$$

so that $v_{\varepsilon}(x, t)$ only goes weakly to zero.

We now investigate the question of the propagation of singularities. Recall that if $u \in \mathscr{D}^{\prime}(\Omega)$, then its wave front set, $W F(u)$, is a subset of $T^{*}(\Omega) \backslash 0$ defined by 


$$
W F(u)=\bigcap\left\{\gamma(A): A u \in C^{\infty}, A \in L_{1,0}^{0}(\Omega)\right\},
$$

where $A$ is a pseudodifferential operator of order zero and $\gamma(A)$ is the set of characteristics of $A$. We refer to [3], [4] for notation and further details. If $\left\{u_{j}\right\}$ is a directed set of distributions, and if $u_{j} \rightarrow u$ in $\mathscr{D}^{\prime}(\Omega)$, we define the wave front set of $\left\{u_{j}\right\}, W F\left\{u_{j}\right\}$, as follows: Call a pseudodifferential operator $A \in L_{1,0}^{0}(\Omega)$ smoothing for $\left\{u_{j}\right\}$ if $A u_{j} \rightarrow A u$ in $C^{\infty}(\Omega)$. Then

$$
W F\left\{u_{j}\right\}=\bigcap\left\{\gamma(A): A \text { is smoothing for }\left\{u_{j}\right\}\right\} \text {. }
$$

A related concept is the following. Let $\Gamma$ be a closed conic subset of $T^{*}(\Omega) \backslash 0$ and let $H_{\Gamma}^{s}(\Omega)=\left\{u \in H^{s}(\Omega): W F(u) \subset \Gamma\right\}$. Then $u \in H^{s}(\Omega)$ if and only if both (i) $u \in H^{s}(\Omega)$, and (ii) $A u \in C^{\infty}(\Omega)$ for each $A \in L_{1,0}^{0}(\Omega)$ whose symbol is of order $-\infty$ on a conic neighborhood of $\Gamma$. Since it suffices for (ii) to hold for a countable set of $A, H^{s}(\Omega)$ can be given a natural topology by means of (i) and (ii) and is thus a Fréchet space. Note that $u_{j} \rightarrow u$ in $H_{\Gamma}^{s}(\Omega)$ implies that $W F\left\{u_{j}\right\} \subset \Gamma$.

Let $\Omega=R^{n}$ and consider the behavior of Fourier integral operators on $H_{\Gamma}^{s}\left(R^{n}\right)$. We shall say that $q \in C^{\infty}\left(R^{n} \times R^{n}\right)$ is in $S_{1,0}^{m}\left(R^{n}\right)$ if $\left|D_{x}^{\beta} D_{\xi}^{\alpha} q(x, \xi)\right| \leqq$ $C_{\alpha, \beta}(1+|\xi|)^{m-|\alpha|}$. Now suppose that $\phi(x, \xi)$ is a nondegenerate phase function, associated with the canonical relation $C$, and consider the Fourier integral operator

$$
I_{q} u(x)=(2 \pi)^{-n} \int q(x, \xi) e^{i \phi(x, \xi)} \hat{u}(\xi) d \xi .
$$

LEMMA 1. For each closed conic subset $\Gamma$ of $T^{*}\left(R^{n}\right) \backslash 0, I_{q}$ maps $H_{\Gamma}^{s}$ into $H_{C \circ \Gamma}^{s-m}$ continuously, and the map $q \rightarrow I_{q}$ from $S_{1,0}^{m}\left(R^{n}\right)$ into $\mathscr{L}\left(H_{\Gamma}^{s}, H_{C \circ \Gamma}^{s}\right)$ is continuous.

Proof. That $I_{q} u \in H_{C \circ \Gamma}^{s-m}$ for all $u \in H_{\Gamma}^{s}$ follows from [4], and the continuity assertions follow easily from the closed graph theorem.

We apply this lemma to the system (2) where $k=2$ and the coefficients are constant.

THEOREM. Consider the initial value problem

(3) $\frac{\partial}{\partial t} u_{\varepsilon}+\sum_{j=1}^{n} A_{j} \frac{\partial}{\partial x_{j}} u_{\varepsilon}=\varepsilon \sum_{k, j=1}^{n} B_{k j} \frac{\partial^{2}}{\partial x_{k} \partial x_{j}} u_{\varepsilon}, \quad \varepsilon>0, u_{\varepsilon}(x, 0)=\Phi(x)$.

We assume that $A_{j}, B_{k j}$ are constant $2 \times 2$ matrices, that the eigenvalues of $\sum \xi_{j} A_{j}$ are real and distinct and that the eigenvalues of $\sum \xi_{j} \xi_{k} B_{j k}$ are positive, both for real $\xi \neq 0$. If $\Phi \in H_{\Gamma}^{s}\left(R^{n}\right)$ and if $C_{t, v}$ are the canonical relations on $T^{*}\left(R^{n}\right) \backslash 0$ determined at time $t$ by the bicharacteristic flows given by the 
hyperbolic operator $L=\partial / \partial t+\sum A_{j} \partial / \partial x_{j}(v=1,2)$, then $u_{\varepsilon}(x, t) \rightarrow u(x, t)$ in $H_{C_{t, 1} \circ \Gamma \cup C_{t, 2}{ }^{\circ} \Gamma}^{s}$.

We outline the proof, where, for simplicity, we take $n=1$. We further suppose $A=A_{1}$ has been put in diagonal form, ${ }^{3} A=-\operatorname{diag}(\alpha, \beta)$, and only consider the case where $B=B_{11}$ is positive definite and symmetric, i.e., $B=\left(\begin{array}{c}a \\ c \\ c\end{array}\right)$, where $a b>c^{2}, a>0$, and $c \neq 0$. Thus, we consider the problem

$$
\frac{\partial}{\partial t} u_{\varepsilon}-\left(\begin{array}{cc}
\alpha & 0 \\
0 & \beta
\end{array}\right) \frac{\partial}{\partial x} u_{\varepsilon}=\left(\begin{array}{ll}
a & c \\
c & b
\end{array}\right) \frac{\partial^{2}}{\partial x^{2}} u_{\varepsilon}, \quad \varepsilon>0, u_{\varepsilon}(x, 0)=\Phi(x) .
$$

The solution is given by

$$
u_{\varepsilon}(x, t)=\frac{1}{(2 \pi)^{1 / 2}} \int_{-\infty}^{\infty} e^{i x \xi} e^{\Delta(t, \xi)} \hat{\Phi}(\xi) d \xi,
$$

where $\Delta=-i t \xi A-\varepsilon t \xi^{2} B$. To evaluate this, we first diagonalize $\Delta$. The eigenvalues of $\Delta$ are

$$
\lambda_{ \pm}=-\frac{1}{2} \varepsilon t \gamma_{1} \xi^{2} \pm \frac{1}{2} t \xi\left(\varepsilon^{2} \gamma_{2}^{2} \xi^{2}-\gamma_{3}^{2}+i \varepsilon \xi \gamma_{4}\right)^{1 / 2}+i t \xi \gamma_{5},
$$

where $\gamma_{1}=a+b, \gamma_{2}^{2}=(a-b)^{2}+4 c^{2}, \gamma_{3}^{2}=(\alpha-\beta)^{2}, \gamma_{4}=-2(a-b)$ $\cdot(\alpha-\beta), \gamma_{5}=(\alpha+\beta) / 2$. Some easy calculations give

$$
e^{\Delta}=S e^{\operatorname{diag}\left(\lambda_{+}, \lambda_{-}\right)} S^{-1}=\frac{1}{u_{+}-u_{-}}\left(\begin{array}{cc}
u_{+} e^{\lambda_{+}}-u_{-} e^{\lambda_{-}} & u_{+} u_{-}\left(e^{\lambda_{-}}-e^{\lambda_{+}}\right) \\
e^{\lambda_{+}}-e^{\lambda_{-}} & u_{+} e^{\lambda_{-}}-u_{-} e^{\lambda_{+}}
\end{array}\right),
$$

where

$$
u_{ \pm}=\left(\lambda_{ \pm}+\varepsilon t \xi^{2}-i t \beta \xi\right) /-\varepsilon t \xi^{2} c .
$$

We consider first the case $\gamma_{4} \neq 0$. Then $u_{ \pm}(\varepsilon, \xi)$ are analytic functions of $\xi$ for all real $\xi$ and $u_{+}-u_{-}$is never zero. The assertion that the wave front set of $u$ propagates along bicharacteristic strips issuing from the wave front set of $\Phi$ follows from

LEMMA 2. The following sets of functions are all bounded in $S_{1,0}^{0}(R)$ : (a) $u_{ \pm}\left(u_{+}-u_{-}\right)^{-1}$, (b) $\left(u_{+}-u_{-}\right)^{-1}$, (c) $u_{+} u_{-}\left(u_{+}-u_{-}\right)^{-1}$, (d) $\exp \left[u_{+}-\right.$it $\left.\alpha \xi\right]$, (e) $\exp \left[u_{-}-\right.$it $\left.\beta \xi\right]$.

The last two are the hardest, so we consider only (d). Set

$$
q_{\varepsilon}(\xi)=\exp \left[u_{+}(\varepsilon, \xi)-i t \alpha \xi\right] ;
$$

we are to show that there exist $C_{j}$ independent of $\varepsilon, \xi$ such that

$$
q_{\varepsilon}^{(j)}(\xi) \leqq C_{j}(1+|\xi|)^{-j}
$$

If we write $q_{\varepsilon}(\xi)=e^{S_{\varepsilon}(\xi)}$, then we have

\footnotetext{
${ }^{3}$ Although this is not necessary, and could not be done if $n>1$.
} 
and

$$
\operatorname{Re} S_{\varepsilon}(\xi) \leqq-\delta \varepsilon \xi^{2}, \quad \text { for some } \delta>0,
$$

$$
q_{\varepsilon}^{(j)}(\xi)=e^{S_{\varepsilon}(\xi)} \sum_{j=1}^{l} \sum_{v_{1}+\cdots+v_{l}=j ; v_{n} \geqq 0} k_{v, j} S_{\varepsilon}^{\left(v_{1}\right)}(\xi) \cdots S_{\varepsilon}^{\left(v_{l}\right)}(\xi),
$$

for some constants $k_{v, j}$; thus we are left with investigating the derivatives of $S_{\varepsilon}(\xi)$. We have

$$
\begin{aligned}
S_{\varepsilon}^{\prime}(\xi)= & -\varepsilon t \gamma_{1} \xi+\frac{1}{2} t\left(\left(\varepsilon^{2} \gamma_{2}^{2} \xi^{2}-\gamma_{3}^{2}+i \varepsilon \xi \gamma_{4}\right)^{1 / 2}-i \gamma_{3}\right) \\
& +\frac{\frac{1}{2} t \gamma_{2}^{2} \varepsilon^{2} \xi^{2}+\frac{1}{4} i t \gamma_{4} \varepsilon \xi}{\left(\varepsilon^{2} \gamma_{2}^{2} \xi^{2}-\gamma_{3}^{2}+i \varepsilon \xi \gamma_{4}\right)^{1 / 2}}, \\
S_{\varepsilon}^{(j)}(\xi)= & \frac{t}{2} \xi \frac{d^{j}}{d \xi^{j}}\left(\varepsilon^{2} \gamma_{2}^{2} \xi^{2}-\gamma_{3}^{2}+i \varepsilon \xi \gamma_{4}\right)^{1 / 2} \\
+ & \frac{j}{2} \frac{d^{j-1}}{d \xi^{j-1}}\left(\varepsilon^{2} \gamma_{2}^{2} \xi^{2}-\gamma_{3}^{2}+i \xi \varepsilon \gamma_{4}\right)^{1 / 2}, \quad j>1,
\end{aligned}
$$

with a term $-\varepsilon t \gamma_{1}$ if $j=2$. Hence we get an estimate of the form

$$
\begin{aligned}
& \left|\frac{d^{j}}{d \xi^{j}}\left(\varepsilon^{2} \gamma_{2}^{2} \xi^{2}-\gamma_{3}^{2}+i \varepsilon \gamma_{4} \xi\right)^{1 / 2}\right| \\
& \quad \leqq C\left|\varepsilon^{2} \gamma_{2}^{2} \xi^{2}-\gamma_{3}^{2}+i \varepsilon \gamma_{4} \xi\right|^{1 / 2-j} \sum_{k=j}^{2 j} \varepsilon^{k}(1+|\xi|)^{k-j}
\end{aligned}
$$

We require

Lemma 3. There exists a $C$ independent of $\varepsilon, \xi$ such that

$$
\left|\varepsilon^{2} \gamma_{2}^{2} \xi^{2}-\gamma_{3}^{2}+i \varepsilon \gamma_{4} \xi\right|^{-1} \leqq C+C \varepsilon|\xi| .
$$

Using this lemma we get

$$
\left|S_{\varepsilon}^{(j)}(\xi)\right| \leqq(1+|\xi|)^{-j}(1+\varepsilon|\xi|)^{j-1 / 2} \sum_{k=j-1}^{2 j} \varepsilon^{k}(1+|\xi|)^{k+1} \quad(j>2)
$$

with a harmless term $C \varepsilon$ thrown in for $j=2$, and, for $j=1$,

$$
\left|S_{\varepsilon}^{\prime}(\xi)\right| \leqq C^{\prime} \varepsilon(1+|\xi|) .
$$

This estimate for $S_{\varepsilon}^{\prime}(\xi)$ is the one crucial place where the term $-i t \alpha \xi$ in the exponential plays a role. Without it, we would merely have $\left|S_{\varepsilon}^{\prime}(\xi)\right| \leqq$ $C+C \varepsilon(1+|\xi|)$ and this is insufficient for (5). If we now use (7) and (8) in (6), we get (5). Next, if $\gamma_{4}=0$ and if $\Phi \in \mathscr{E}^{\prime}(R)$, we can write

$$
u(x, t)=\frac{1}{(2 \pi)^{1 / 2}} \int_{-\infty}^{\infty} e^{i x(\xi+i \tau)+i t(\xi+i \tau)-\varepsilon t(\xi+i \tau)^{2} A} \hat{\Phi}(\xi+i \tau) d \xi,
$$

where $\tau$ is fixed and chosen so that $\left(\varepsilon^{2} \gamma_{2}^{2}(\xi+i \tau)^{2}-\gamma_{3}^{2}\right)^{1 / 2}$ does not vanish. 
The rest of the argument goes through without change except that Lemma 3 must be replaced by the following weaker estimate

$$
\left|\varepsilon^{2} \gamma_{2}^{2}(\xi+i \tau)^{2}-\gamma_{3}^{2}\right|^{-1} \leqq C+C \varepsilon|\xi|^{2} .
$$

However, this is still enough to prove (5).

We complete the proof of the Theorem. Set $A_{\varepsilon}(\Phi)=u_{\varepsilon}$. Lemma 2 shows that we can write $A_{\varepsilon}=A_{1, \varepsilon}+A_{2, \varepsilon}$ with $\left\{A_{v, \varepsilon}: 0<\varepsilon \leqq 1\right\}$ a bounded subset of $I^{0}\left(R^{n}, C_{t, v}\right)$, the space of Fourier integral operators of order zero, with the topology induced by the set of symbols. From Lemma

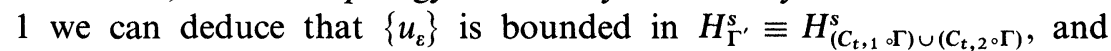
moreover that $u_{\varepsilon} \rightarrow u$ in $H_{\Gamma^{\prime}}^{s}$.

ACKNowledgment. The authors are grateful to Carl de Boor for his kind assistance with the numerical calculations.

\section{REFERENCES}

1. Charles C. Conley and Joel A. Smoller, Viscosity matrices for two-dimensional nonlinear hyperbolic systems, Comm. Pure Appl. Math. 23 (1970), 867-884. MR 43 \# 714.

2. J. J. Duistermaat and Lars Hörmander, Fourier integral operators. II, Acta Math. 128 (1972), 183-269.

3. Lars Hörmander, On the existence and the regularity of solutions of linear pseudodifferential equations, Enseignment Math. (2) 17 (1971), 99-163.

4. —_ Fourier integral operators. I, Acta Math. 127 (1971), 79-183.

5. Peter D. Lax, Asymptotic solutions of oscillatory initial value problems, Duke Math. J. 24 (1957), 627-646. MR 20 \# 4096.

Department of Mathematics, University of Michigan, Ann Arbor, Michigan 48104 